\title{
Die Macht der Philologie oder: Wie man mit Goethes freundlicher Unterstützung Polen germanisieren wollte
}

\author{
Von Robert Rduch
}

1.

Bei der Sichtung der Stoffe für postkoloniale Studien in der deutschen Germanistik wird häufig die Kolonisierung im osteuropäischen Zusammenhang übersehen. Die Tatsache, dass der Kampf gegen Germanisierung einen festen Topos der polnischen Literatur und folglich ein relevantes Thema in der polnischen Geisteswissenschaft bereits vor der Etablierung postkolonialer Theorien bildete, wurde in der deutschen Forschung lange Zeit schlicht ignoriert ${ }^{1}$. Das mag verständlich sein, wenn man bedenkt, dass eine vorurteilsfreie Erkundung deutsch-polnischer Beziehungen durch die politische Atmosphäre des 20. Jahrhunderts noch bis 1989 beeinträchtigt worden war. Die Entwicklung der germanistischen Literaturwissenschaft in Deutschland in den letzten dreißig Jahren zeigt jedoch, dass deutsche Geisteswissenschaftler die Geschichte der Beziehungen zum östlichen Nachbarn als Forschungsfeld postkolonialer Studien sehr langsam und mit großer Mühe entdecken ${ }^{2}$.

In einem überblicksartigen Beitrag von Gabriele Dürbeck über die Entwicklung postkolonialer Ansätze in der deutschen und internationalen Germanistik wird zwar betont, dass ,die Aufarbeitung der vernachlässigten Kolonialgeschichte und ihrer Zeugen in Literatur, Medien und Alltagskultur ${ }^{663}$

1 Selten greifen deutsche Forscher auf Werke von Henryk Sienkiewicz, Bolesław Prus und Maria Konopnicka zurück. In den zwei ersten deutschen Einführungen zur postkolonialen Theorie wurden sowohl die Rolle der Literaturwissenschaft in Deutschland als auch die Relevanz der Theorie für die deutsche Kultur nur in geringem Ausmaß berücksichtigt, ganz zu schweigen von einem Hinweis auf koloniale Verhältnisse in Europa. Vgl. Maria do Mar Castro Varela, Nikita Dhawan: Postkoloniale Theorie. Eine kritische Einführung. Bielefeld: transcript 2005 (2., komplett überarbeitete Auflage 2015); Ina Kerner: Postkoloniale Theorien zur Einführung. Hamburg: Junius 2012.

2 Die deutsche Forschung erhielt wichtige Anregungen in dieser Hinsicht aus Amerika. Vgl. Christoph Kienemann: Der koloniale Blick gen Osten. Osteuropa im Diskurs des Deutschen Kaiserreiches von 1871. Paderborn: Ferdinand Schöningh 2018, S. 10.

3 Gabriele Dürbeck: Deutsche und internationale Germanistik. In: Dirk Göttsche, Axel Dunker, Gabriele Dürbeck (Hg.): Handbuch Postkolonialismus und Literatur. Stuttgart: Metzler 2017, S. 38-53, hier S. 39. 
den Kern dieser Forschungen bildet, aber Osteuropa findet in dem Überblick keine Erwähnung. Binnenkolonialismus wird in dem Handbuch Postkolonialismus und Literatur als wissenschaftliche Entdeckung des 21. Jahrhunderts wahrgenommen: „Erst in einem zweiten Schritt hat sich die Aufmerksamkeit der postkolonialen Forschung seit den späten 1990er Jahren auch auf den europäischen Binnenkolonialismus gerichtet, der den Übersee-Kolonialismus historisch jedoch von Anfang an begleitet und ergänzt hat. ${ }^{\prime 4}$ Die Autoren des Handbuches verweisen in dieser Hinsicht zu Recht auf die Arbeiten von Kristin Kopp, aber das opulente Werk Text-Ränder. Die kulturelle Vielfalt in Mitteleuropa als Darstellungsproblem deutscher Literatur ${ }^{5}$ von Jürgen Joachimsthaler wurde in dem Kompendium nicht einmal bibliographisch erfasst.

Der aktuelle Forschungsstand zwingt also nicht nur zur Fokussierung auf Zeugnisse des kolonialen Denkens in Deutschland, sondern auch auf den Umgang der Wissenschaftler und Publizisten mit diesen Zeugnissen.

Die Begriffe Macht und Gewalt bestimmen das koloniale Denken und sie beziehen sich auch auf die Wissenschaft. Unter Berufung auf den indischen Forscher Dipesh Chakrabarty verweist Ina Kerner darauf, dass die kritische Beleuchtung des Zusammenhangs zwischen Macht und Wissen zu den wichtigsten Forschungspostulaten der postkolonialen Auseinandersetzung mit der Vergangenheit gehört ${ }^{6}$. Dabei wird deutlich, dass sich in den Machtstrukturen der Kolonisatoren „Strategien der Beschwichtigung unliebsamer kolonialer Ereignisse und Erfahrungen ${ }^{67}$ herausbildeten.

Ich will hier im wörtlichen Sinne auf ein Archiv zurückgreifen und Strategien des Beschweigens im Bereich der Literaturgeschichte im Zusammenhang mit der gewaltsamen Verbreitung der deutschen Sprache in Europa

4 Epp Annus, Marijan Bobinac, Dirk Göttsche, Julia-Karin Patrut: Europäischer Binnenkolonialismus in interdisziplinärer Perspektive. In: Dirk Göttsche, Axel Dunker, Gabriele Dürbeck (Hg.): Handbuch Postkolonialismus und Literatur. Stuttgart: Metzler 2017, S. 87-96, hier S. 87.

5 Vgl. Jürgen Joachimsthaler: Text-Ränder. Die kulturelle Vielfalt in Mitteleuropa als Darstellungsproblem deutscher Literatur. Bd. 1. Schreibweisen; Bd. 2 (Post-)Koloniale Textur; Bd. $3 »$ Dritte Räume«. Heidelberg: Winter 2011. Vgl. auch Robert Rduch: Text-Ränder im (post)kolonialen Diskurs oder was man ,aus sich hinausborussifizieren muss“. In: http:// www.kakanien-revisited.at/rez/RRduch2.pdf (19.4.2019).

6 Vgl. Ina Kerner: Postkoloniale Theorien zur Einführung. Hamburg: Junius 2012, S. 152.

7 Ebd., S. 150. Kerner bezieht sich hier auf das Buch Silencing the Past (1995) des haitanischen Anthropologen Michel-Rolph Trouillot. 
verfolgen. Germanisierung als ein Teilaspekt der Kolonisierung ist in erster Linie mit Sprachpolitik verbunden. Der Versuch, einer ethnischen Gruppe eine fremde Sprache aufzuzwingen, wird in den postkolonialen Studien als wichtiges Instrument der epistemischen Gewalt aufgefasst. Zur Etablierung dieser sprachlichen Gewalt wurden Kunst und Wissenschaft herangezogen. Am Beispiel des Aufsatzes Vorschlag zur Einführung der deutschen Sprache in Polen, eines Textes, dessen Autorschaft Goethe zugeschrieben wird, sowie der publizistischen und wissenschaftlichen Rezeption des Aufsatzes versuche ich, die Rolle der Philologie bei der Förderung, Verschleierung und Aufdeckung des kolonialen Denkens zu analysieren.

\section{3.}

Der Aufsatz Vorschlag zur Einführung der deutschen Sprache in Polen wurde zum ersten Mal 1892 im Goethe-Jahrbuch von Bernhard Suphan veröffentlicht. Suphan zweifelte nicht an der Autorschaft des Textes, obwohl sich aus den erhaltenen Manuskripten ergibt, dass nur einige Korrekturen und die letzten Sätze von Goethes Hand stammen. Der Titel sowie der Untertitel „Um eine höhere Cultur der niederen Classen zu bewirken" wurden von Eckermann hinzugeschrieben ${ }^{8}$. Suphan datierte den Text auf die Jahre 1793-17959.

Der Text enthält ein fremdsprachendidaktisches Konzept: Die deutsche Sprache sollte durch das Theater vermittelt werden. Wandernde Theatertruppen bringen dem polnischen Volk nicht nur die wichtigsten Wörter und Wendungen bei, sondern auch eine höhere Kultur, „die gebildetere Sitte der herrschenden Nation" ${ }^{\prime \prime}$, aus der sich lebenspraktische Vorteile ergeben. Das didaktische Konzept steht in dem Aufsatz deutlich im Zusammenhang mit einem politischen und militärischen Konflikt, der nach der Besitznahme des polnischen Territoriums als „,ein innerer Krieg ${ }^{\text {"111 }}$ fortdauert, da „der eroberte Staat von dem erobernden an Sprache und Sitte verschieden ist" ${ }^{" 12}$.

Der Kommentar des Herausgebers ist nicht auf Details der Editionsphilologie eingeschränkt. Suphan las den Aufsatz durch die Brille der wilhelminischen Politik und betrachtete ihn als eine willkommene Stütze der Germanisierung. Der erste Satz seines Kommentars lautet:

8 Vgl. Vorschlag zur Einfürung der deutschen Sprache in Polen. Ein unbekannter publicistischer Versuch Goethes. In: Goethe-Jahrbuch Bd. 13 (1892), S. 3-9, hier S. 3.

9 Ebd., S. 8.

10 Ebd., S. 5.

11 Ebd., S. 3.

12 Ebd. 
„Als deutscher Culturkämpfer im wahren Sinne des Wortes, das heisst als Vorkämpfer für vaterländische Sprache und Sitte und deren Verbreitung zeigt sich unser Goethe hier seinen Verehrern und Freunden von einer neuen Seite."13

Suphan bezeichnete den Text als die ,zu rechter Stunde unter den Schätzen des Goethe-Archivs gefunden[e] Abhandlung ${ }^{\text {"14 }}$.

Hier wurde das Gebiet der Philologie verlassen, Goethe wurde nicht mehr als Gegenstand einer wissenschaftlichen Analyse betrachtet, sondern als ein nationales Symbol der Deutschen. Der nationalisierte Klassiker wurde zu einem Instrument im Prozess der Kolonisierung der deutschen Ostmark ${ }^{15}$.

Der aus dem Archiv zutage geförderte Text bereitete sowohl deutschen als auch polnischen Philologen und Publizisten große Probleme. Sie entwickelten drei Interpretationsstrategien:

a) Einige wenige Interpreten, es handelt sich ausschließlich um deutsche Wissenschaftler und Publizisten, akzeptierten die Deutung von Suphan und begrüßten Goethes Aufsatz als Bestätigung der aktuellen Politik, ohne den Inhalt des Aufsatzes und dessen Entstehungsgeschichte genauer zu untersuchen.

b) Eine größere Gruppe sieht Goethes Text kritisch. Hier überwiegen polnische Interpreten. Goethes Haltung im Aufsatz wurde von ihnen entschieden verurteilt.

c) Die größte Gruppe besteht aus Goethes Verteidigern, es sind nicht nur Deutsche, sondern auch Polen. Die ersteren wollten die unbefleckte Humanität des nationalen Klassikers retten, die letzteren dachten an den Klassiker der Weltliteratur und an die deutsch-polnischen Beziehungen. Es wurde nach Rechtfertigungsargumenten gesucht. Von vielen wurde der Aufsatz einfach verschwiegen.

13 Bernhard Suphan: [Kommentar]. In: Ebd., S. 8.

14 Ebd.

15 Suphan war in der wilhelminischen Ära einer der vielen Philologen und Literaten, die die deutsche Kultur für die Kolonisierung der preußischen Provinzen mit polnischer Bevölkerung nutzten. Vgl. dazu das Kapitel „Die Ostmarkenliteratur“ in: Jürgen Joachimsthaler: Text-Ränder. Die kulturelle Vielfalt in Mitteleuropa als Darstellungsproblem deutscher Literatur. Bd. 1. Schreibweisen. Heidelberg: Winter 2011, S. 190-204. 
Bei meiner Recherche habe ich mehr als 50 Texte über Goethes Vorschlag oder Texte, in denen ich eine Stellungnahme zu Goethes Aufsatz vermutete, darunter direkte Besprechungen der Erstveröffentlichung, spätere synthetische Darstellungen der Beziehung des Dichters zu Polen sowie Publikationen zum Bild Polens in der deutschen Literatur, untersucht. Im Folgenden werde ich nur ausgewählte Beispiele der Rezeption des Vorschlags, gegliedert in die oben genannten drei Gruppen, präsentieren, u.a. mit dem Ziel, typische Beschwichtigungskonzepte des kolonialen Denkens sowie antikoloniale Abwehrmaßnahmen zu veranschaulichen.

5.

Die Entdeckung des unveröffentlichten Aufsatzes von Goethe fand unter deutschen Philologen und Publizisten kein großes Echo. Suphans Deutung konnte nur wenige überzeugen. In einer der ersten deutschen Reaktionen auf die Erstveröffentlichung 1892 schrieb Albert Pick, Rezensent einer Posener Zeitschrift: „Auf jeden Fall ist diese Veröffentlichung dankenswerth, in welcher sich unser Goethe - um mit dem Herausgeber zu reden - »als Vorkämpfer für vaterländische Sprache und Sitte und deren Verbreitung « zeigt." ${ }^{16}$ Die Solidarität des Rezensenten mit dem Herausgeber des Aufsatzes bestätigt die Kontinuität des kolonialen Denkens in Deutschland seit den Teilungen Polens bis zum Kulturkampf in der zweiten Hälfte des 19. und zu Beginn des 20. Jahrhunderts. Der Rezensent vertrat mit einem Wort den Standpunkt des Kolonisators auf dem kolonisierten Gebiet.

Dass die deutsche Philologie und Publizistik dem Vorschlag jedoch bis zum Ende des 1. Weltkrieges wenig Aufmerksamkeit schenkte, bezeugte George von Gravenitz im Goethe-Handbuch von 1918. Er modifizierte allerdings die Auslegung des nationalen Engagements von Goethe und nannte eine andere Entstehungszeit, ohne sie zu begründen: „Wenig bekannt ist, daß Goethe der 'polnischen Frage' in ihrem besonderen Bezug auf den Schutz der deutschen Sprache in polnischem Sprachgebiet einen besonderen Aufsatz 'Vorschlag zur Einführung der deutschen Sprache in Polen' gewidmet hat. Er bezieht sich auf die in der sog. vierten polnischen Teilung an Preußen gekommenen Landesteile

16 Albert Pick: Vorschlag zur Einführung der deutschen Sprache in Polen. Ein unbekannter publicistischer Versuch Goethes. In: Zeitschrift der Historischen Gesellschaft für die Provinz Posen 7 (1892), S. 358-359, hier S. 359. 
und ist wahrscheinlich nach 1813-1814 gedruckt worden." ${ }^{\text {"17 }}$ Sechsundzwanzig Jahre nach der Erstveröffentlichung aus dem Archiv ging es also nicht mehr um Verbreitung, sondern um ,den Schutz der deutschen Sprache“. Mit dieser Interpretation antizipierte Gravenitz bereits deutsche Gebietsverluste nach dem 1. Weltkrieg.

Kritische Reaktionen auf die Veröffentlichung des unbekannten Textes von Goethe ließen nicht lange auf sich warten. Einen der ersten Kommentare schrieb der Feuilletonredakteur der „Neuen Freien Presse“ Ludwig Speidel. Er bewertete den Vorschlag aus österreichischer Perspektive als ,einen naiv lehrhaften Vortrag ${ }^{\alpha 18}$. Ironisch erklärte er, dass die einzelnen Völker in der habsburgischen Monarchie gar nicht die deutsche Sprache annehmen wollen, und gab zu, dass der Vorschlag eine Gewaltaktion darstellte: „Vollends ein Volk, das, gleich den Polen, gewaltsam unter fremde Botmäßigkeit gerathen ist, wird die Aufforderung, die Sprache der Gewaltherren anzunehmen, nicht anders aufnehmen, als die Zumutung, seine eigene Seele aufzugeben" ${ }^{\text {"19 }}$. Mit diesen Worten verurteilte er indirekt die koloniale Politik der Deutschen im Osten.

Das Urteil polnischer Publizisten fiel noch deutlicher aus. Man lehnte nicht nur Goethes didaktische Idee ab, sondern attackierte auch Suphans philologische Leistung. Eine der ersten polnischen Reaktionen erschien am 12. Mai 1892 in „Gazeta Lwowska“ [Lemberger Zeitung]. Ein anonymer Berichterstatter informierte über eine Ausgrabung aus dem, so wörtlich, „Archivmüll““ [,ze śmieci archiwalnych" ${ }^{\text {“20] }}$ und vermutete, der große Dichter würde sich für die Veröffentlichung schämen. Die vorsichtige Kritik an Goethe wurde hier mit einem Hinweis auf seine späteren Kontakte zu Polen aufgewogen. Eine ausführliche Besprechung des Vorschlags für das polnische Publikum lieferte der bekannte Historiker Szymon Askenazy 1893 in der Zeitschrift „Biblioteka Warszawska“. Als Liebhaber des deutschen Dichters kritisierte

17 George von Gravenitz: Polen. In: Julius Zeitler (Hg.): Goethe-Handbuch. Bd. 3. Stuttgart: J. B. Metzlersche Verlagsbuchhandlung 1918, S. 143-144. Gravenitz übernahm die Entstehungszeit von Oskar Walzel. Vgl. Johann Wolfgang Goethe: Sämtliche Werke: Jubiläums-Ausgabe in 40 Bänden. Bd. 37: Schriften zur Literatur. Teil 2. Stuttgart [u.a.]: Cotta 1906, S. 33-36.

18 L. Sp. [Ludwig Speidel]: Allerlei Goethe. In: Neue Freie Presse vom 3.7.1892, S. 1-3, hier S. 1.

19 Ebd.

20 [anonym]: Goethe germanizatorem Polaków. In: Gazeta Lwowska vom 12.05.1892, S. 3. Alle Übersetzungen polnischer Texte in dem vorliegenden Artikel stammen von R.R. 
er die Verwendung des Aufsatzes zur Propagierung der deutschen Sprache. Er übersetzte mehrere Passagen des Vorschlags ins Polnische und meinte, dass sich Goethe durch die Formulierung einer solchen pädagogischen Idee lächerlich gemacht habe. Die Philologen, die den Aufsatz mit einem politischen Kommentar veröffentlichten, wurden von ihm verspottet: „Was wird damit nachgewiesen? Dass der große Dichter in einem schlechteren Moment eine Lächerlichkeit beging. Diese Lächerlichkeit hat er zum Glück nicht im Druck verewigt, er warf sie in den Papierkorb, den sie nicht mehr verlassen sollte. Aber wozu gibt es Monographisten, insbesondere deren vorzüglichste Art, die sich Goetheforscher nennt?“"21.

1898 veröffentlichte Albert Zipper ${ }^{22}$ in der Zeitschrift „Kraj“ einen kritischen Artikel unter dem Titel Goethe germanizatorem Polski [Goethe als Germanisierer Polens], der beim polnischen Publikum das negative Bild Goethes für Jahre prägte ${ }^{23}$. Zipper rekapitulierte die wichtigsten Fakten über Goethes Beziehung zu Polen und kam zu dem Schluss, dass die Veröffentlichung des Vorschlags zur Revision der Auffassung, Goethe sei ein Freund Polens, führen muss. Er referierte den Inhalt des Aufsatzes, übersetzte kurze Abschnitte ins Polnische und deutete Goethes Idee als einen Versuch ,das polnische Element zu vernichten “24. Noch stärker als den Klassiker kritisierte er die antipolnische Tendenz der Philologen, die den Vorschlag publiziert hatten, obwohl er kindisch sei und im Gegensatz zu Goethes Leben und Werk stehe. Die Philologen, von Zipper als „Fanatiker eines Goethe-Kults“25 apostrophiert, wollten in dem Vorschlag keine Schwäche des Dichters sehen, sondern den Nachweis einer patriotischen Haltung.

Sachlich und kritisch präsentierte Goethes Vorschlag Stanisław Leonhard 1912 in der Krakauer Zeitschrift „Wiedza i Postęp“. Der Publizist führte mehrere Episoden aus dem Leben des Dichters an, um nachzuweisen, dass ihm Polen gleichgültig war. Leonhard betonte, dass man Goethes Aufsatz in Polen nicht bagatellisieren dürfe und erinnerte daran, dass die Germanisierung im preußischen Teilungsgebiet (Großpolen) nach wie vor intensiv betrieben

21 Szymon Askenazy: J. W. Goethe, Projekt do zaprowadzenia języka niemieckiego w Polsce. In: Biblioteka Warszawska 1893, Nr. 4, S. 152-154, hier S. 154.

22 Albert Zipper (1855-1936) war Germanist, Übersetzer und Lexikograph aus Lemberg. Vgl. Jacek D. Iciaszek: Albert Zipper. In: Christoph König (Hg.): Internationales Germanistenlexikon 1800-1950. Bd. 3: R-Z. Berlin, New York: Walter de Guyter 2003, S. 2109-2111.

23 Vgl. Edmund Kołodziejczyk: Goethe w Polsce. Rzecz literacko-bibliograficzna. Kraków: Fundusz Naukowy, Drukarnia i Stereotypia Eugeniusza i Dra Kazimierza Koziańskich 1913, S. 4, 10. In der Bibliographie von Kołodziejczyk fungiert der Artikel von Zipper als die einzige polnische Besprechung des Vorschlags. Kołodziejczyks bibliographische Angaben sind falsch.

24 Albert Zipper: Goethe germanizatorem Polski. In: Kraj Jg. 17 (1898), Nr. 6, S. 67-68, hier S. 67.

25 Ebd., S. 67. 
werde. In diesem aktuellen Kontext betrachtete er den deutschen Dichter als Vorläufer der kolonialen Politik im wilhelminischen Deutschland: „Goethe zeigte den Deutschen heute, auf welchem Wege man in dem neu erworbenen Land Kultur verbreiten soll.“" ${ }^{\text {'6 }}$ Einen eindeutig kritischen Standpunkt vertraten auch Edmund Kołodziejczyk und Henryk Mościcki, die die Argumente von Zipper und Leonhard wiederholten ${ }^{27}$.

Die polnische Debatte um den Vorschlag entbrannte erneut 1929 nach einem Artikel von Jan Obst, in dem Goethe als „,der erste Hakatist“"28 bezeichnet wurde, weil er mit seinem didaktischen Projekt richtungsweisend auf die Germanisierungspolitik im deutschen Kaiserreich gewirkt habe. Obst sah in dem pädagogischen Konzept die Überheblichkeit der deutschen Kulturträger der ihnen unbekannten polnischen Kultur gegenüber und konnte nicht verstehen, wie der große Dichter die Missachtung ,der heiligsten Schätze: der Muttersprache, der Kultur, der Eigenart, der Sitten der Väter“29 in Polen in Kauf nehmen konnte.

Ein ähnlich eindeutiges Urteil wurde von einem deutschen Germanisten erst $1970 \mathrm{im}$ Kommentar der Berliner Ausgabe von Goethes Werken formuliert. Siegfried Seidel skizzierte den historischen Kontext der Teilungen Polens, verwies auf Goethes Abneigung den polnischen Unabhängigkeitsbestrebungen gegenüber und betrachtete dessen pädagogisches Konzept als Ausdruck imperialistischen Denkens. Auch wenn man bei der Lektüre des Kommentars daran denkt, dass die Geschichtswissenschaft in der DDR in den ideologischen Kampf gegen den Westen eingespannt war, erscheint die Argumentation Seidels mit den Grundsätzen heutiger postkolonialer Theorien vereinbar:

„Goethe billigt somit die preußische Eroberungspolitik, wenn er auch, wie der „Vorschlag“"zeigt, mit „,eineren“ Mitteln vorgehen möchte, die jedoch nicht weniger nationalistisch und reaktionär sind. Es verwundert durchaus nicht, da $\beta$ Bernhard Suphan in der Wilhelminischen Ära schreiben konnte: ,Als deutscher Kulturkämpfer im wahren Sinne des Wortes, d.h. als Vorkämpfer für vaterländische Sprache und Sitte und deren Verbreitung zeigt sich unser Goethe hier seinen Verehrern und Freunden von einer neuen Seite.' Diese „,neue Seite“ war

26 Stanisław Leonhard: Stosunek Goethego do Polski. In: Wiedza i Postęp. Czasopismo poświęcone popularyzacji wiedzy i zdobyczy techniki Jg. 1 (1912), Nr. 10, S. 273-283, hier S. 280.

27 Vgl. Edmund Kołodziejczyk: Goethe w Polsce. Rzecz literacko-bibliograficzna. Kraków: Fundusz Naukowy, Drukarnia i Stereotypia Eugeniusza i Dra Kazimierza Koziańskich 1913; Henryk Mościcki: Stosunek Goethego do Polski. In: Tygodnik Ilustrowany 1915, Nr. 7, S. 100.

28 Jan Obst: A jednak Goethe nie byt przyjacielem Polaków. In: Polonia Jg. 6 (1929), Nr. 1668, S. 3. 'Hakatist' war die polnische Bezeichnung für Mitglieder des Deutschen Ostmarkenvereins.

29 Ebd. 
unterdessen von großbürgerlichen Eroberungspolitikern ausreichend erprobt, zur Unterdrückung anderer Nationen ausgenutzt und zu einem Grundprinzip der imperialistischen Politik erhoben worden. Gerade weil die reaktionären Kräfte im 19. und 20. Jahrhundert andere Völker nicht nur mit Waffengewalt unterwarfen, sondern ihnen auch die eigene Sprache und Kultur aufzwangen, ist es notwendig, auf die noch immer gefährliche politisch-ideologische Konsequenz eines solchen „Vorschlags“" hinzuweisen.“30

Einen der wenigen eindeutig kritischen Kommentare zum Vorschlag in der polnischen Germanistik nach 1945 publizierte Jan Papiór erst 1991. Er opponierte gegen Versuche, Goethes Standpunkt zu rechtfertigen. Papiór beurteilte den Aufsatz im Zusammenhang mit anderen Aussagen des Dichters und betonte die Notwendigkeit einer historischen Kontextualisierung des Vorschlags: „J. W. Goethe war mit seinem Vorschlag weder der Erste noch der Letzte. Die Deutschen werden heute das beschämende Kapitel aus der Geschichte der deutschen Bildung nicht ans Licht bringen wollen.“31

In dieser Hinsicht irrte er sich, denn die allmähliche Etablierung kulturwissenschaftlicher Konzepte in der deutschen Literaturwissenschaft begünstigte die Auseinandersetzung mit Goethes Vorschlag. Er wurde in Arbeiten sowohl deutscher als auch polnischer Germanisten im Kontext postkolonialer Theorien berücksichtigt. Man widmete ihm allerdings nicht viel Aufmerksamkeit und es entsteht der Eindruck, als ob Forscher im 21. Jahrhundert davon ausgehen können, dass der Vorschlag bereits ausführlich kommentiert worden ist. Izabela Surynt erwähnte Goethes Abhandlung im Kontext des deutschen Diskurses über die neuen preußischen Gebiete nach den Teilungen Polens. Die Verbindung mit kolonialen Diskursen war für die Autorin selbstverständlich:

„Die Imaginierung des östlichen Nachbarn und - nicht zu vergessen - der eigenen Mitbürger als, Wilde' und ,Barbaren' oder ,Indianer' speist sich somit einerseits aus den semantischen Implikationen des modernen Fortschrittsund Kulturbegriffes sowie der immer wieder aktualisierten asymmetrischen Einteilung und Wahrnehmung der Menschheit; andererseits lassen sich in ihr Reflexe des Kolonialdiskurses (und zwar sowohl der deutschen Kolonial- und Kolonisationspläne als auch der Kolonialismuskritik) herausstellen. Die etwas ältere Attribuierung des polnischen ,Nationalcharakters' als ,barbarisch und ,wild' sowie der ,moderne' Vergleich der polnischen Gesellschaft mit den amerikanischen Indianerstämmen erlaubte zugleich die Aufstellung von diversen Integrationsprojekten den neuen Mitbürgern gegenüber, wie z.B. das

30 Siegfried Seidel: Vorschlag zur Einführung der deutschen Sprache in Polen. In: Johann Wolfgang Goethe: Werke. Bd. 17: Kunsttheoretische Schriften und Übersetzungen. Schriften zur Literatur I. Berlin: Aufbau 1970, S. 858-861, hier S. 860.

31 Jan Papiór: Kontekst historyczny poloniców J. W. Goethego. Rozważania na marginesie rocznicy śmierci pisarza. In: Neofilolog Kujawski 1 (1991), S. 81-101, hier S. 94. 
Konzept von Goethe Vorschlag zur Einführung der deutschen Sprache in Polen (1795/96) oder das von Karl Grashoff aus dem Jahre 1796 Einige Ideen zur Beantwortung der Frage: Wie lässt sich die Bildung einer Nation am leichtesten und sichersten auf eine andere übertragen? Mit beständiger Hinsicht auf die gegenwärtige Teilung von Polen zur Überprüfung und weiteren Ausführung. Das hier entwickelte Alteritätssystem wird die Wahrnehmung der polnischen Kultur bis in das 20. Jahrhundert hinein grundlegend prägen." 32

Während in den Text-Rändern von Jürgen Joachimsthaler der Vorschlag als Beispiel für innere Kolonisation nur kurz erwähnt wurde ${ }^{33}$, glaubten Alfred Sproede und Mirja Lecke Goethes Abhandlung für postkoloniale Studien entdeckt zu haben: „Ein vielleicht unerwartetes, aber umso deutlicher autoritatives Belegstück für die Berechtigung solcher Studien kommt von: Johann Wolfgang von Goethe. Der deutsche Groß-Klassiker hat die Polonophobie seiner Zeit (im Geiste Voltaires und anderer ,Stipendiaten' Katharinas II.) um eine neue, gleichsam administrative Wendung bereichert, die in der Polonistik nicht gebührende Aufmerksamkeit gefunden hat, obwohl die Parallele zu einer ,kolonialistischen' Auffassung der deutsch-polnischen Beziehungen auf der Hand liegt. ${ }^{\text {" }} 4$ Die Verwunderung der Autoren über das schwache Interesse der Polonistik an Goethes Vorschlag ist verständlich, aber auf dem Forschungsfeld der deutsch-polnischen Beziehungen sind Germanisten und Historiker kompetenter. Sproede und Lecke übersahen auch die Tatsache, dass der Vorschlag sowohl Polonisten als auch Germanisten bereits zu Beginn des 20. Jahrhunderts bekannt war und im Diskurs über deutsch-polnische Beziehungen deutliche Spuren hinterließ.

Die scheinbar selbstverständliche Kritik am Vorschlag zur Einführung der deutschen Sprache in Polen war aber nicht das dominierende Deutungsmuster in

32 Izabela Surynt: Polen als Raum des, Anderen' am Beispiel der deutschsprachigen Literatur der 1820er und der 1830er Jahre. In: Alfred Gall, Thomas Grob, Andreas Lawaty, German Ritz (Hg.): Romantik und Geschichte. Polnisches Paradigma, europäischer Kontext, deutsch-polnische Perspektive. Wiesbaden: Harrassowitz 2007, S. 295-310, hier S. 297.

33 Vgl. Jürgen Joachimsthaler: Text-Ränder. Die kulturelle Vielfalt in Mitteleuropa als Darstellungsproblem deutscher Literatur. Bd. 1. Schreibweisen. Heidelberg: Winter 2011, S. 81.

34 Alfred Sproede, Mirja Lecke: Der Weg der postcolonial studies nach und in Osteuropa. Polen, Litauen, Russland. In: Dietlind Hüchtker, Alfrun Kliems (Hg.): Überbringen Überformen - Überblenden. Theorietransfer im 20. Jahrhundert. Köln, Weimar, Wien: Böhlau 2011, S. 27-66, hier S. 35. 
der Auseinandersetzung mit dem Aufsatz. In vielen Fällen, wenn man Goethes Beziehungen zu Polen thematisierte, wurde der unbequeme Aufsatz einfach totgeschwiegen oder man versuchte, die Haltung des Dichters zu rechtfertigen.

1907 publizierte Józef Tretiak den Artikel Goethe i Hakata ${ }^{35}$. Der Titel legt nahe, dass der Autor Stellung nimmt zu Goethes Vorschlag, aber in dem Artikel wurde dieser nicht einmal erwähnt. Stattdessen versuchte Tretiak nachzuweisen, dass man die letzten Szenen im zweiten Teil des Faust als eine Warnung vor den Folgen gewaltsamen Kolonisierens lesen kann. Er widmete seine Interpretation den Mitgliedern des Deutschen Ostmarkenvereins. Leonhard kritisierte Tretiak dafür, dass er Goethes Tragödie als antikoloniale Kritik im Kontext der deutschen Kolonisierungspolitik in Großpolen präsentierte, ohne in dem Artikel den Vorschlag zu berücksichtigten ${ }^{36}$. Von einer Strategie des Verschweigens ist auch der Beitrag Polacy $i$ Goethe von Władysław Bełza geprägt. Das kolonisatorische Konzept des Dichters wurde nicht genannt, obwohl Bełza seinen Artikel Albert Zipper widmete, der 1898 Goethe als Germanisierer Polens bezeichnet hatte. Auch in Artikeln, die auf Deutsch publiziert wurden, blieb Goethes Vorschlag unerwähnt ${ }^{37}$.

Diese Vermeidungsstrategie löste 1929 in Polen eine Debatte aus, die bis zum 100. Todestag von Goethe dauerte. Sie wurde hervorgerufen durch Eugen Mellers Ausführungen Czy Goethe byt przyjacielem Polaków? ${ }^{38}$ [War Goethe ein Freund der Polen?], in denen der Autor auf die Erwähnung des Vorschlags verzichtete. Darauf reagierte der schon genannte Jan Obst, der die Frage negativ beantwortete und den großen Dichter, wie gesagt, als Hakatisten bezeichnete ${ }^{39}$. Die Attacke auf Goethe versuchte Spiridion Wukadinović, Germanistikprofessor an der Krakauer Jagiellonen-Universität ${ }^{40}$, mit zwei Publikationen abzuwehren. Er verkleinerte die Bedeutung des Konzeptes von

35 Vgl. Józef Tretiak: Goethe i Hakata. In: Czas Nr. 296 vom 24.12.1907, S. 1-2.

36 Vgl. Stanisław Leonhard: Stosunek Goethego do Polski. In: Wiedza i Postęp. Czasopismo poświęcone popularyzacji wiedzy i zdobyczy techniki. Jg. 1 (1912), Nr. 10, S. 282-283.

37 Vgl. Bertold Merwin: Polnische Literatur (Goethes und Hebbels Beziehungen zu Polen - Ergebnisse letzter Forschungen). In: Österreichische Rundschau Jg. 35 (1913), S. 154-158; Eugen Meller: Goethes Beziehungen zu Polen. In: Österreichische Rundschau Jg. 37 (1915), S. 44-46; Josef Nadler: Goethe und der deutsche Osten. In: Corona 1939, Nr. 6, S. 655-674; Ludwig Goldstein: Goethe und Ostpreußen. In: Ostdeutsche Monatshefte Jg. 11 (1930), Nr. 7, S. 407-412.

38 Vgl. Eugen Meller: Czy Goethe byt przyjacielem Polaków? Garść odkryć archiwalnych w Krakowie, Frankfurcie, Weimarze i Karlsbadzie. In: Polonia Jg. 6 (1929), Nr. 1658, S. 17-18.

39 Vgl. Jan Obst: A jednak Goethe nie był przyjacielem Polaków. In: Polonia Jg. 6 (1929), Nr. 1668, S.3.

40 Vgl. Maria Kłańska: Spiridion Wukadinović (1870-1938). In: Wojciech Kunicki, Marek Zybura (Hg.): Germanistik in Polen. Zur Fachgeschichte einer literaturwissenschaftichen Auslandsgermanistik - 18 Porträts. Osnabrück: fibre 2011, S.17-32. 
Goethe, weil „es unveröffentlicht mit anderen Papieren liegen blieb und erst 60 Jahre nach Goethes Tode bekannt wurde, ohne je aktuell geworden zu sein ${ }^{641}$. Wukadinović behauptete, dass Goethes Kritiker im Kontext der schlechten deutsch-polnischen Beziehungen zusätzlich „Haß und Zwietracht säen“442. Damit missachtete er den Kontext der Erstveröffentlichung des Vorschlags und übersah auch die koloniale Dimension des ,kleinen, harmlosen Aufsatzes“443. Allen, die hinter dem Titel Vorschlag zur Einführung der deutschen Sprache in Polen „ein hochpolitisches Manifest ${ }^{\star 44}$ vermuteten, erklärte Wukadinović: „Umso angenehmer ist man enttäuscht, wenn man sieht, wie der Dichter hier mit friedlichen und vornehmen Mitteln erzielen will, was die Machthaber dieser Erde nur durch Gewaltmaßnahmen erzwingen zu können vermeinen. Wer fühlte sich da nicht an die bekannte Fabel vom Wettstreit des Windes und der Sonne erinnert, wem es eher gelingen werde, dem Wanderer den Mantel zu entreissen? Der Sturm lässt alle seine Künste spielen, aber der Wanderer hüllt sich nur noch dichter in den schützenden Umhang. Da bricht die Sonne mit ihren wärmenden Strahlen hervor, und zufrieden lächelnd legt er den Mantel ab. ${ }^{645}$

Die meisten Kommentatoren des Streits verharmlosten Goethes kolonisatorische Pläne, rechtfertigten seine Haltung mit dem Hinweis auf seinen Kosmopolitismus $^{46}$ oder verzichteten in ihren Beiträgen zum 100. Todestag des Dichters auf die Erwähnung des Vorschlags ${ }^{47}$. Selbst kurz vor dem deutschen Angriff auf Polen 1939 wurde in der „Zeitschrift für Geschichte und Gegenwart des Ostdeutschtums“ die Humanität des Vorschlags verteidigt:

41 Spiridion Wukadinović: Goethe - »der erste Hakatist«. In: Deutsche Blätter in Polen Jg. 6 (1929), Nr. 8, S. 379-382, hier S. 380.

42 Ebd., S. 382.

43 Spiridion Wukadinović: Goethe und Polen. Aus Anlass der Goethe-Ausstellung in Danzig (Oktober 1930). Gdańsk: Towarzystwo Przyjaciół Nauki i Sztuki w Gdańsku 1930, S. 16-17.

44 Ebd., S. 17.

45 Ebd., S. 17-18.

46 Vgl. Zygmunt Łempicki: Die Goethe-Literatur in Polen. In: Ostdeutsche Monatshefte Jg. 11 (1930), Nr. 7, S. 461-463: „Der im Goethe-Jahrbuch, Band 13 (1892), veröffentlichte Vorschlag Goethes, 'Zur Einführung der deutschen Sprache in Polen', hat selbstverständlich in Polen großes Interesse erweckt (vgl. z. B. A. Zipper im 'Kraj', 1897) und Anlaß zu heiklen Erörterungen über Goethes Stellung zu Polen gegeben.“; Hermann Sternbach: Spiridion Wukadinović: Goethe und Polen. In: Ruch Literacki Jg. 6 (1931), Nr. 4, S. 121-122; Michał Friedländer, Jan Piprek: Poktosie rocznicy śmierci Goethego w Polsce. In: Neofilolog Jg. 3 (1932), Nr. 3, S. 135-142; Julian Twardowski: Stosunek Goethego do narodu polskiego. In: Czas Jg. 84 (1932), Nr. 71, S. 2.

47 Vgl. Samuel Stendig: Na marginesie poktosia rocznicy Goethego w Polsce. In: Neofilolog Jg. 3 (1932), S. 198-199; Bronisław Gubrynowicz: Kilka notatek o Goethem w Polsce. In: Ruch Literacki Jg. 7 (1932), Nr. 3, S. 68-77; Hermann Sternbach: Goethe a Polska. In: Filomata 1932, Nr. 42, S. 5-11. 
„Man hat in Polen selbst früher gern eine polenfeindliche Stellung Goethes feststellen wollen und zur Erhärtung dieser These in erster Linie eine spätgefundene fremde Niederschrift aus dem Nachlaß des Dichters herangezogen, die unter dem mißverstandenen Titel, Vorschlag zur Einführung der deutschen Sprache in Polen' fernab jeder Tagespolitik lediglich den humanen Zielen des deutschen Theaters hat dienen wollen und praktisch niemals - auch für den Dichter nicht $-\mathrm{zu}$ einer besonderen Geltung gelangt ist. So werden heutzutage auch die größten Eiferer eine These „Goethe der erste Hakatist" nicht mehr verteidigen können. ${ }^{، 48}$

Auch nach dem 2. Weltkrieg ist die Rezeption des Aufsatzes von Goethe durch Beschweigen und Rechtfertigungsversuche dominiert. Antoni Trepiński erinnerte 1947 in einer Anthologie an den Kommentar von Szymon Askena$z^{49}$. Jan Wypler übersetzte 1949 mehrere Passagen aus dem Aufsatz, aber er verharmloste zugleich die Wirkung des Vorschlags ${ }^{50}$. Einen philologisch und rhetorisch misslungenen Versuch, Goethe zu verteidigen, stellen zwei Publikationen des Warschauer Germanisten Michał Cieśla ${ }^{51}$ dar. 1961 äußerte er sich über den Vorschlag, ohne den Aufsatz gelesen zu haben. Er behauptete, „in der größten Ausgabe der gesammelten Werke des Dichters ist der Artikel nicht vorhanden ${ }^{\text {“52 }}$. Deshalb basierte er nur auf dem Vortrag Goethe und Polen des Krakauer Germanisten Wukadinović und kopierte dessen Argumentation, indem er die Milde des Germanisierungsprojekts von Goethe betonte. 1962 machte Cieśla keinen Hehl daraus, dass er mit seinem Beitrag Goethe rehabilitieren und an ähnliche Versuche aus dem Jahre 1932 anknüpfen möchte ${ }^{53}$. Inkonsequenterweise versuchte er aber mehrmals anzudeuten, dass man das

48 Robert Adolf Klostermann: Goethe und Polen. In: Deutsche Monatshefte. Zeitschrift für Geschichte und Gegenwart des Ostdeutschtums Jg. 6 (1939/1940), Nr. 1, S. 34-37, hier S. 36 .

49 Vgl. Antoni Trepiński (Hg.): Potępieńcy. Sąd wieków nad Niemcami. Antologia. Łódź: Ksiegania Ludowa T. Lemański 1947, S. 67.

50 Vgl. Jan Wypler: Goethe. Podróżnik po Polsce-Miłośnik polskich kobiet-Germanizator $i$... Weltbuerger. In: Odra Jg. 5 (1949), Nr. 11, S. 2.

51 Vgl. Karol Sauerland: Michat Cieśla. In: Wojciech Kunicki, Marek Zybura (Hg.): Germanistik in Polen. Zur Fachgeschichte einer literaturwissenschaftlichen Auslandsgermanistik - 18 Porträts. Osnabrück: fibre 2011, S. 227-237.

52 Michał Cieśla: Język polski w szkołach berlińskich w latach 1797-1807. In: Rozprawy z Dziejów Oświaty 1961, Nr. 4, S. 87-124, hier S. 89. Cieśla erklärte nicht, welche Ausgabe er meint. Goethes Aufsatz wurde bereits 1907 in die Weimarer Ausgabe aufgenommen. Vgl. Johann Wolfgang Goethe: [Vorschlag zur Einführung der deutschen Sprache in Polen]. In: Goethes Werke hrsg. im Auftrage der Großherzogin Sophie von Sachsen. Bd. 42. Zweite Abtheilung. Weimar: Hermann Böhlaus Nachfolger 1907, S. 18-23.

53 Michał Cieśla: Goethe a pruska polityka germanizacyjna w Polsce. In: Przegląd Humanistyczny 1962, Nr. 2, S. 141-145, hier S. 141-142. 
kolonisatorische Konzept nicht rechtfertigen könne ${ }^{54}$. Bei der Erwähnung von polnischen Attacken gegen Goethe nannte er kein einziges Beispiel, auch seine Erklärungen zur Entstehungszeit des Vorschlags sind keine Zeugnisse philologischer Sorgfalt ${ }^{55}$. Nach Cieśla verfasste Goethe den Vorschlag unter dem Einfluss antipolnischer Publizistik und ,im Glauben daran, dass Germanisierung der unterdrückten Nation dienen wird. "56

Mangelndes Wissen kommt nicht in Frage im Falle von zwei Artikeln in deutscher Sprache, die in den 1960er Jahren erschienen. Den Titeln nach, Polen und Johann Wolfgang Goethe ${ }^{57}$ sowie Goethe und Polen ${ }^{58}$, könnte man annehmen, dass in diesen Texten das Germanisierungskonzept wenigstens erwähnt wurde. In beiden Artikeln entschieden sich die Autoren jedoch für eine Vermeidungsstrategie.

Einen ernsthaften Versuch, Goethe von dem Vorwurf zu entlasten, er habe die Germanisierung Polens unterstützt, unternahm 1980 Erich Trunz. Der kompetente Goethe-Forscher legte die bisher beste philologische Analyse des Vorschlags vor und stellte Goethes Autorschaft des Aufsatzes in Frage. Seinen Standpunkt begründete er sachlich, und zum Schluss bemerkte er: „Wie die vorstehenden Ausführungen zeigen, habe ich keinen handfesten Beweis, um diesen Aufsatz Goethe abzusprechen, etwa eine Erwähnung in einem Brief oder dergleichen. Doch Suphan hatte ebensowenig einen sicheren Beweis, ihn Goethe zuzusprechen. Da nun der eine Philologe den Aufsatz Goethe zugesprochen hat und der andere ihn ihm abspricht, schlage ich vor, ihn als 'zweifelhaft' zu bezeichnen. "'59

Geradezu bezeichnend für die Tabuisierung des Vorschlags unter polnischen Germanisten sind zwei Publikationen von dem Breslauer

54 Vgl. ebd., S. 142: „Wie auch immer wir diesen Auftritt des Dichters bewerten, man darf ihn auf keinen Fall rechtfertigen, aber man kann ihn andererseits auch nicht einer Feindschaft gegen Polen und Slawen im Allgemeinen, eines Nationalismus oder Chauvinismus bezichtigen.“; S. 145: „Abschließend dürfen wir ihn nicht völlig rechtfertigen, aber wir können ihn verstehen."

55 Vgl. ebd., S. 143: Cieśla gab bei seiner Behauptung, Suphan zweifelte an der Autorschaft des Textes, keine Quelle an. An einer anderen Stelle schrieb Cieśla: „Nach Suphans Vermutungen wurde der Text gegen 1794 geschrieben, eigentlich Eckermann diktiert.“ Im Jahre 1794 war Johann Peter Eckermann zwei Jahre alt!

56 Ebd.

57 Vgl. Norbert Honsza: Polen und Johann Wolfgang Goethe. In: Deutsch-polnische Hefte 5 (1962), Nr. 2, S. 61-69; Nr. 3, S. 137-139.

58 Vgl. Hermann Buddensieg: Goethe und Polen. In: Mickiewicz-Blätter 13 (1968), Nr. 37, S. 1-30.

59 Erich Trunz: An den Grenzen des Goetheschen Werkes. Zuschreibungen, Abschreibungen, Zweifelhaftes, unzureichend Ediertes. In: Ders.: Weimarer Goethe-Studien. Weimar: Hermann Böhlaus Nachfolger 1980, S. 85-110, hier S. 98-99. Das Unterkapitel, in dem Goethes Vorschlag behandelt wurde, hat den Titel „Ein sehr zweifelhafter Aufsatz“ (S. 92-99). 
Literaturwissenschaftler Marian Szyrocki, der 1980 in der Zeitschrift „,Germanica Wratislaviensia“ den Artikel Goethe und Polen veröffentlichte. Er fasste das Wissen über die Beziehungen des Dichters zu Polen zusammen, nannte aber nicht einmal den Titel des Aufsatzes über die didaktische Benutzung des Theaters bei den Germanisierungsbestrebungen ${ }^{60} .1981$ veröffentlichte Szyrocki ein Buch über Leben und Werk von Goethe auf Polnisch. Auf über dreihundert Seiten schilderte er den künstlerischen Werdegang des Dichters. Sichtlich erleichtert informierte er den polnischen Leser auf der letzten Seite seiner Monographie: „Und doch lastete auf Goethes Haltung unserer Nation gegenüber ein Schatten. In einer der Schriften soll er eine Idee der Germanisierung Polens dargestellt haben. Die Tatsache, dass die Schrift nicht aus Goethes Feder stammte, bestätigte vor kurzem mit großer Wahrscheinlichkeit Erich Trunz."61 Auch hier verschwieg Szyrocki den Titel dieser Schrift.

Einer ähnlichen Vermeidungsstrategie folgten die Herausgeber und Autoren des „Germanistischen Jahrbuches DDR - VRP“, das 1982 dem Thema der „Wechselbeziehungen zwischen Polen und Goethe und der Rezeption, die das Schaffen Goethes von den Anfängen bis zur Gegenwart in Polen gefunden hat, ${ }^{\text {"“62 }}$ gewidmet war. Die im Voraus bestimmte Richtung der Reflexion über Goethes Werk erschwerte die Auseinandersetzung mit dem Thema, denn man wollte „,den völkerverständigenden tieferen Humanismus eines Großen der Weltliteratur noch einmal deutlich werden [...] lassen ${ }^{\text {"63. }}$. Mit einem so formulierten Prinzip wurde die philologische Arbeit nicht weiterentwickelt, sondern durch Perpetuierung ideologiekonformer Denkschemata gelähmt. In dem Beitrag Goethe in der polnischen Literaturkritik und Literaturforschung bis $1939^{64}$ von Tadeusz Namowicz wurde Goethes Vorschlag nicht erwähnt. Erst in dem Artikel Zur Goethe-Rezeption in Polen von Edyta Połczyńska wurde der Germanisierungsidee des Dichters eine Seite gewidmet ${ }^{65}$. Die Autorin nannte in diesem Zusammenhang nur den Vortrag Goethe und Polen von Wukadinović sowie den Artikel Goethe a pruska polityka germanizacyjna w Polsce von Cieśla, dem sie sonderbarerweise eine hohe analytische Qualität attestierte. Die Erkenntnisse der Herausgeber und Autoren des Jahrbuches blieben also weit

60 Vgl. Marian Szyrocki: Goethe und Polen. In: Germanica Wratislaviensia 1980, Nr. 40, S. 81-98.

61 Marian Szyrocki: Johann Wolfgang Goethe (2. Auflage). Warszawa: Wiedza Powszechna 1987, S. 326.

62 Horst Ziebart: Zum Geleit. In: Germanistisches Jahrbuch DDR - VRP (1980/1982), S. 3-4, hier S. 4.

63 Ebd.

64 Vgl. Tadeusz Namowicz: Goethe in der polnischen Literaturkritik und Literaturforschung bis 1939. In: Germanistisches Jahrbuch DDR - VRP (1980/1982), S. 5-23.

65 Vgl. Edyta Połczyńska: Zur Goethe-Rezeption in Polen. In: Germanistisches Jahrbuch DDR - VRP (1980/1982), S. 24-39, hier S. 28-29. 
hinter dem Kommentar der Berliner Ausgabe sowie der kritischen Publizistik aus der ersten Hälfte des 20. Jahrhunderts zurück.

Die Rechtfertigungsversuche der Goethe-Philologie erreichten einen grotesken Höhepunkt 1986 in der Münchner Goethe-Ausgabe. Wahrscheinlich völlig unbewusst sanktionierte Klaus H. Kiefer die kolonisatorische Botschaft des Aufsatzes von Goethe unter Verwendung eines Begriffes, den er der postkolonialen Politik in der zweiten Hälfte des 20. Jahrhunderts entnommen hatte. Er erläuterte das Germanisierungskonzept im Kontext der antirevolutionären Atmosphäre in Deutschland am Ende des 18. Jahrhunderts als „Entwicklungshilfe“: „G., der vor machtpolitischen Realismen an sich nicht zurückschreckte (G. zu F. v. Müller, i. Januar 1832), skizziert dagegen ein relativ unkriegerisches Entwicklungshilfe-Modell im Sinne soziokultureller Evolution. Dieses Modell ist vor dem Hintergrund einer dem Lande durchaus nicht unvorteilhaften preußischen Sprach- und Schulpolitik zu sehen, die freilich die Eroberung polnischen Gebietes erst einmal voraussetzt und auf die politische Integration der Bevölkerung abzielt.“66

Die beste Kontextualisierung des Vorschlags lieferte Gerard Koziełek 1989 mit seiner Anthologie Das Polenbild der Deutschen 1772-1848, in der Goethes Aufsatz neben anderen Texten aus der Epoche publiziert und im Zusammenhang mit der „Diskussion über die geistige Annexion der polnischen Bevölkerung ${ }^{667}$ erläutert wurde. Koziełek beleuchtete die preußische „Kolonisationspolitik“68 deutlich und veranschaulichte sie mit mehreren Beispielen, so dass Goethes Germanisierungskonzept eindeutig als Ausdruck eines kolonisatorischen Denkens erscheint. Trotzdem war auch Koziełek um eine Rechtfertigung des Dichters bemüht: „Goethe gedachte seine Ansicht zu diesem Thema zu veröffentlichen, unterließ aber schließlich dieses Ansinnen; zweifellos wurde er sich des Antihumanen der von ihm unterstützten Kulturpolitik bewußt."69 Mit einem ähnlichen Kommentar versah Koziełek die Erwähnung des Vorschlags in einem polnischen Lexikon ${ }^{70}$.

Die Goethe-Philologie der letzten zwanzig Jahre erzielte keine Fortschritte hinsichtlich des Vorschlags. Der diesbezügliche Kommentar in der Frankfurter Ausgabe der Werke von Goethe ähnelt eher einer Impression des Herausgebers:

66 Kommentar zu [Vorschlag zur Einführung der deutschen Sprache in Polen]. In: Johann Wolfgang Goethe: Sämtliche Werke nach Epochen seines Schaffens. Bd. 4.2. Wirkungen der Französischen Revolution 1791-1797. München: Hanser 1986, S. 937-939, hier S. 938.

67 Gerard Koziełek: Das Polenbild der Deutschen 1772-1848. In: Ders. (Hg.): Das Polenbild der Deutschen 1772-1848. Anthologie. Heidelberg: Carl Winter 1989, S. 11-70, hier S. 22.

68 Ebd., S. 21.

69 Ebd., S. 22.

70 Gerard Koziełek: Niemiecko-polskie zwiazki literackie. In: Józef Bachórz, Alina Kowalczykowa (Hg.): Stownik literatury polskiej XIX wieku. Wrocław 2009, S. 608-612, hier S. 609 . 
Es wird in ihm auf die Sonderbarkeit des präsentierten Konzeptes verwiesen, auf dessen antirevolutionären Charakter angespielt und von der Hauptidee abgelenkt, statt sie historisch und philologisch zu erläutern. Es heißt dort: „Angesichts von Goethes Kritik am Nationalismus und seines Desinteresses am preußischen Staat, mag dieser gleichsam imperialistische, die Eroberung Polens sanktionierende Beitrag sonderbar erscheinen. Er dürfte vor dem Hintergrund jakobinischer Umtriebe in Polen antirevolutionär motiviert sein. Interessanter ist aber die hier entwickelte Vorstellung von kultureller Evolution. ${ }^{\text {“71 }}$ Friedmar Apel erklärte nicht, welche „kulturelle Evolution“ und welche „Umtriebe“ er meinte. Auch die Goethe-Handbücher scheinen den bisherigen Forschungsstand sehr selektiv zu verwerten. In Gero von Wilperts Kommentar scheint Goethe um die Lösung eines preußischen Problems bemüht zu sein: „Die drei polnischen Teilungen von 1772, 1793 und 1795 und der polnische Aufstand 1830/31 hinterlassen in seinem Werk kaum Spuren. Nur ein um 1795 entstandener, erst postum gedruckter Aufsatz 'Vorschlag zur Einführung der deutschen Sprache in Polen' nimmt auf das Sprachproblem der Preußen angegliederten Landesteile Polens Bezug, das durch Aufführungen erzieherisch beispielhafter deutscher Familiendramen durch deutsche Schauspielertruppen gemildert werden könnte. ${ }^{\text {"72 } I m ~ G o e t h e-H a n d b u c h ~}{ }^{73}$ und im Metzler-Goethe-Lexikon ${ }^{74}$ wurde Goethes Vorschlag übergangen.

8.

Wie dieser Einblick in die Geschichte der Rezeption des Vorschlags zur Einführung der deutschen Sprache in Polen zeigt, hat die Philologie in der Konfrontation mit dem Aufsatz versagt. Mehrere Autoren, die sich über Goethes Beziehung zu Polen äußerten, übergingen den einzigen Text des Dichters mit einem deutlichen Bezug zu Polen nicht nur aus Ignoranz, sondern auch aus der Furcht, eine Erörterung des Aufsatzes könnte dem Ansehen des Dichters

71 Friedmar Apel: [Kommentar zu] Vorschlag zur Einführung der deutschen Sprache in Polen. In: Johann Wolfgang Goethe: Sämtliche Werke. Briefe, Tagebücher und Gespräche. Bd. 18. Ästhetische Schriften 1771-1805. Hrsg. von Friedmar Apel. Frankfurt am Main: Deutscher Klassiker Verlag 1998, S. 1184.

72 Gero von Wilpert: Polen. In: Ders.: Goethe-Lexikon. Stuttgart: Krönder 1998, S. 833.

73 Vgl. Hans-Dietrich Dahnke, Regine Otto (Hg.): Goethe-Handbuch. Bd. 4/2. Personen. Sachen. Begriffe L-Z. Stuttgart, Weimar: Metzler 1998. Ein Stichwort ,Polen' gibt es in dem Handbuch nicht.

74 Vgl. Benedikt Jeßing: Polen. In: Bendikt Jeßing, Bernd Lutz, Inge Wild (Hg.): Metzler-Goethe-Lexikon: Personen - Sachen-Begriffe. Stuttgart, Weimar: Metzler 2004, S. 339. Der Vorschlag wurde von Jeßing nicht erwähnt. 
oder den deutsch-polnischen Beziehungen einen Schaden zufügen. Goethe ist bis heute auch für viele Literaturwissenschaftler ,ein Erinnerungsort" geblieben, denn er wurde im Zusammenhang mit dem Vorschlag zumeist nicht als ein Objekt wissenschaftlicher Untersuchung, sondern als nationales oder europäisches Symbol betrachtet. Die Stellungnahmen zum Vorschlag verdeutlichten jedoch, dass im Text eine eindeutig koloniale Haltung vertreten wird. Da sich eine solche Haltung mit Goethes sogenannter philologisch idealisierter Humanität kaum in Einklang bringen lässt, versuchten viele Philologen und Publizisten ihn zu rechtfertigen, statt einen Widerspruch im Bild des Dichters zu akzeptieren. Manche Anstrengungen der Rechtfertiger fallen grotesk aus und transportierten das koloniale Denken ins 21. Jahrhundert. Nicht eine idealisierte Menschlichkeit, sondern politische, ästhetische und pädagogische Diskurse vom Ende des 18. Jahrhunderts sowie weitere Archivrecherchen sollten zukünftig die Grundlage für die Erläuterung des Goetheschen Aufsatzes bilden. 